\title{
DISCUSSÃO DOS PRECEITOS DA ATENÇÃO PRIMÁRIA À SAÚDE E DIREITOS HUMANOS EM UMA ATIVIDADE EXTENSIONISTA DE GRADUAÇÃO
}

\section{DISCUSSION OF PRECEPTS OF PRIMARY HEALTH CARE AND HUMAN RIGHTS IN AN UNDERGRADUATE EXTENSION ACTIVITY}

\section{Ubirajara Amaral Vinholes Filho ${ }^{1}$ \\ Déborah Silveira König ${ }^{2}$ \\ Denise Silva da Silveira ${ }^{3}$ \\ Nádia Spada Fiori ${ }^{4}$}

RESUMO

Este trabalho trata de uma discussão sobre dois casos acompanhados durante o estágio curricular do curso de Medicina no que tange aos conceitos de Atenção Primária à Saúde (APS) e suas intersecções com a Declaração Universal dos Direitos Humanos (DUDH). Sua elaboração dá-se pelacrença de que os preceitos e diretrizes da APS são ferramentas essenciais para possibilitar um existir ao usuário de acordo com o que é pregado pela DUDH. Para tanto, usou-se uma abordagem qualitativa em uma série de dois relatos de casos obtidos a partir da vivência de estágio trimestral de dois alunos de medicina em uma Unidade Básica de Saúde (UBS), Escola vinculada à Universidade Federal de Pelotas (UFPel). Após apresentação, exposição da evolução e discussão dos casos, conclui-se que os princípios da APS foram aplicados, de forma a proporcionar um cuidado integral, multiprofissional e qualificado aos pacientes, apesar de alguns entraves logísticos.

Palavras chave: Atenção primária à saúde. Direitos humanos.

\footnotetext{
1 Aluno do curso de Medicina na Universidade Federal de Pelotas, estagiário do Programa de Ensino pelo Trabalho (PET) desde de maio 2016, ex-integrante e vice-presidente da Liga Acadêmica de Medicina da Comunidade e Epidemiologia (LAMCEP). E-mail: biravinholes_06@outlook.com

2 Aluna do curso de Medicina na UFPel, extensionista e integrante da Liga Acadêmica de Medicina da Comunidade e Epidemiologia (LAMCEP), atualmente no cargo de presidente.

E-mail: deborah_konig@hotmail.com

${ }^{3}$ Doutora em Epidemiologia pela UFPel. Técnica em Educação no Departamento de Medicina Social da Faculdade de Medicina da UFPel. E-mail: denisilveira@uol.com.br

${ }^{4}$ Doutora em Epidemiologia pela Universidade Federal de Pelotas. Professora assistente do Departamento de Medicina Social da Universidade Federal de Pelotas. E-mail: nsfiori@yahoo.com.br
} 
This work is about a discussion about two cases accompanied during a curricular internship of the Medicine course in the tangent to the Health Primary Care (HPC) concepts and their intersections with the described at the Universal Declaration of Human Rights (UDHR). Its elaboration happens by the belief that the precepts and HPC guidelines are essential tools to enable an existence to the user according to the preach at the UDHR. Therefore, a qualitative approach was used in a series of two cases reports obtained from de quarterly internship experience of two medical students in a Scholar Basic Health Unit (BHU), linked with the Universidade Federal de Pelotas (UFPel). After cases presentation, exhibition and evolution, it was conclude that the experiences applied the HPC principles, providing a integral, multiprofessional and quality care to the patients, despite some logistical obstacles.

Keywords: Primary health care. Human rights.

\section{INTRODUÇÃO}

Os Direitos Humanos (DH) surgiram em decorrência das atrocidades vividas durante a II Guerra Mundial, promovidas pela experiência nazista (CARVALHO, 2017). Eles foram delineados e adotados pela Organização das Nações Unidas (ONU) em 1948, através da Declaração Universal dos Direitos Humanos (DUDH) (MATTA; CARVALHO, 2017). Os DH são um conjunto de leis e preceitos para que todos os indivíduos do mundo tenham uma vida digna e plena em sociedade, que devem ser seguidos não somente pelos cidadãos, mas também pelos Estados (CARVALHO, 2017; MATTA, 2009; ORGANIZAÇÃO DAS NAÇÕES UNIDAS, 2009). No artigo $25^{\circ}$ da DUDH, versa-se que "Toda a pessoa tem direito a um nível de vida suficiente para lhe assegurar e à sua família a saúde e o bem-estar, [...]", e que "A maternidade e a infância têm direito a ajuda e a assistência especiais". É parte do dever do Estado brasileiro propiciar tais cuidados para sua população (BRASIL, 1988; CARVALHO, 2017; MATTA, 2009; ORGANIZAÇÃO DAS NAÇÕES UNIDAS, 2009).

Para tanto, em 1978, durante a I Conferência Internacional sobre Cuidados Primários em Saúde, em Alma-Ata, os países membros propuseram a política "Saúde para Todos no Ano 2000", que tinha a meta de atingir o maior nível de saúde possível até o ano de 2000 (OMS, 1988). A Declaração de Alma-Ata foi assinada por 134 países, entre eles o Brasil, concordando que a Atenção Primária à Saúde (APS) seria a ferramenta mais adequada para atingir as metas de saúde propostas (ALMA-ATA, 1988; STARFIELD, 2002; FACCHINI, 2008; MATTA, 2009). Neste encontro foi especificado que seus componentes fundamentais eram: educação 
em saúde; saneamento ambiental, especialmente de águas e alimentos; programas de saúde materno-infantis, inclusive imunizações e planejamento familiar; prevenção de doenças endêmicas locais; tratamento adequado de doenças e lesões comuns; fornecimento de medicamentos essenciais; promoção de boa nutrição; e medicina tradicional (ALMA-ATA, 1988).

O conceito da APS foi primeiro aventado como forma de organização do sistema de saúde no Relatório Dawson (MATTA, 2009). Este documento foi promulgado pelo governo inglês, em 1920, e tinha a proposta de contrapor-se ao modelo Flexineriano estadunidense, o qual foi embasado em um modelo de saúde reducionista biológico, na atenção individual e especialista de medicina, gerando um sistema de elevado custo, crescente complexidade e baixa resolutividade (STARFIELD, 2002; MATTA, 2009). Mais recentemente, Starfield propôs um modelo que divide o sistema de saúde em três níveis de cuidado: centros de saúde primários e secundários; serviços suplementares e domiciliares; e hospitais-escola (2002). Os centros de saúde primários e os serviços domiciliares devem ser regionalizados e com a maior parte de sua demanda resolvida por médicos generalistas e, para os casos cuja resolução não fosse possível nesse nível de atenção, haveria os centros de saúde secundários e os hospitais, com atenção especializada, como referência para tais usuários (STARFIELD, 2002; BRASIL, 2012; SIRENA, 2016).

Ainda segundo Starfield (2002), a definição operacional da APS é composta por quatro atributos essenciais: (1) primeiro contato do acesso do usuário ao serviço de saúde: ser acessível e de utilização para o cuidado de agravos novos ou novos episódios de agravos crônicos, exceto urgências e emergências; (2) longitudinalidade: atenção continuada, com utilização do serviço ao longo do tempo, demonstrando e dependendo de uma relação de confiança entre profissionais de saúde e usuário; (3) integralidade: serviços prestados com objetivo de fornecer integralmente saúde ao indivíduo, desde o processo de saúde-adoecimento até os serviços de média e alta complexidade; (4) coordenação da atenção: continuidade do cuidado do indivíduo por um mesmo profissional ou por um bom registro, além da integração e coordenação entre os serviços de saúde (BRASIL, 2010; SIRENA, 2016). Igualmente, identifica três atributos derivados: (1) orientação e atenção à saúde da família: considerar o papel potencial do contexto familiar como parte do cuidado ou do adoecimento; (2) orientação comunitária: reconhecimento das necessidades em saúde da comunidade e planejamento de medidas de melhoramentos e avaliação conjunta dessas; (3) competência cultural: adaptação do serviço com a realidade cultural e social do usuário, facilitando a comunicação e a relação com o serviço (BRASIL, 2010; STARFIELD, 2002).

Conforme os conceitos trazidos acerca da APS, e entendo-a como ferramenta sine qua non para possibilitar um existir digno ao cidadão de acordo com o pregado pela DUDH, este trabalho visa discutir alguns preceitos pre- 
FILHO, U.A.V et. al. - 152

sentes na prática junto à comunidade dos alunos da Universidade Federal de Pelotas (UFPel). Serão abordados dois relatos de caso de usuários adstritos ao território de uma Unidade Básica de Saúde (UBS), que trabalha nos princípios da APS, ocorridos durante o estágio curricular dos alunos de Medicina de Família e Comunidade.

\section{MATERIAIS E MÉTODOS}

O trabalho terá uma abordagem qualitativa e contará com uma sequência de relatos de casos obtidos a partir da vivência de estágio de dois alunos de medicina na Unidade Básica de Saúde (UBS) Areal Leste da UFPel. Após a descrição dos relatos, eles serão discutidos a partir de uma revisão bibliográfica em consonância aos preceitos da APS e, entendendo a saúde como parte constituinte fundamental dos direitos humanos, as relações que estabelecem com estes princípios.

Os dois casos que serão relatados foram colhidos no período de abril a junho de 2016. Eles foram selecionados como forma de avaliação do estágio de Medicina de Família e Comunidade do curso de Medicina e sob a orientação dos preceptores do serviço. Não haverá exposição de dados que possibilitem a identificação dos indivíduos e suas histórias, assim como o nome dos envolvidos será fictício. A escolha destes deu-se por conveniência, uma vez que foram os que mais apresentaram elementos para discussão dos preceitos da APS dentre todos os casos acompanhados.

O ambiente onde o acompanhamento ocorreu foi uma UBS de ensino vinculada à UFPel que se caracteriza como um serviço de APS há mais de três décadas. A UBS está inserida em uma área urbana periférica da cidade de Pelotas, abrangendo uma população de por volta de oito mil usuários. Sua estrutura física conta com uma sala de espera, uma recepção, uma sala de vacina, uma sala de pronto-atendimento/procedimentos de enfermagem, uma farmácia, oito consultórios médicos, uma sala de assistência social, uma sala de orientação acadêmica, uma cozinha, dois banheiros e pátio. A equipe conta com seis médicos, três enfermeiras, uma assistente social, duas nutricionistas, um farmacêutico, seis técnicos de enfermagem, em média seis profissionais de ensino superior e a equipe de agentes comunitários de saúde. Além destes profissionais, conta com os graduandos dos cursos de medicina, enfermagem, nutrição, serviço social e farmácia. 
Romualdo, de 72 anos. Branco, casado, atualmente aposentado, tendo em alguns meses a renda suplementada com a venda de peixes que pesca.

O primeiro contato com o paciente foi no início do estágio, sendo ele encaminhado de um pronto-atendimento (PA) com a enfermagem devido a uma elevação nos níveis tensionais averiguada em controle rotineiro de Tensão Arterial (TA) realizada desde Janeiro de 2016. Além do mais, vale ressaltar um hemoglicoteste (HGT) de $373 \mathrm{mg} / \mathrm{dL}$ com presença interrogada de cetonas; circunferência abdominal de $120 \mathrm{~cm}$; peso de $101 \mathrm{~kg}$, significando um Índice de Massa Corporal (IMC) de 35,4; com demais achados dentro dos limites de referência. Neste primeiro momento, por tratar-se de uma elevação de TA assintomática, a conduta foi expectante, agendando consulta em dois dias.

No encontro agendado, após revisão de prontuário, foi notada presença de síndrome metabólica - Diabetes Mellitus do tipo II (DM II) há 15 anos; Hipertensão Arterial Sistêmica (HAS) há 20 anos, com mau controle tensional; dislipidemia mista há 20 anos; obesidade há 20 anos, naquele momento grau II -; epilepsia há 65 anos, sem crise convulsiva há 25 anos; doença arterial coronariana (DAC) há 10 anos, com história de um cateterismo coronariano há 10 anos e uma Cirurgia de Revascularização Miocárdica (CRM) há 02 anos; e artrite gotosa, no momento, em fase aguda. Sobre suas medicações, tratava-se do que a literatura define como polifarmácia contando com 13 medicações, dentre elas anti-hipertensivos, antidiabéticos orais, anticonvulsivantes, psicofármacos, Inibidor da Bomba de Prótons (IBP) e redutores de ácido úrico (RAMOS et al., 2016).

\section{Caso 2}

Nize, mulher, 30 anos. Parda, portadora de diabetes tipo I (DM I), tabagista, dona de casa, que reside com dois outros filhos e o marido, o qual sustenta as despesas da casa.

Trata-se de uma usuária gestante, realizando pré-natal (PN) de sua quinta gestação, com história de um aborto, três nascimentos de filhos vivos por via vaginal, sendo que um faleceu aos três meses de idade em decorrência que complicações respiratórias, em 2006.

A atual gestação não foi planejada, mas segundo a paciente foi bem aceita pelo companheiro e familiares, embora a quantificação de beta-HCG tenha sido solicitada para reiniciar a anticoncepção. Após a detecção da gravidez, os exames do primeiro trimestre foram solicitados e ela prontamente encaminhada à referência de alto risco para acompanhamento pré-natal devido ao DM I (BRASIL, 2010; BRASIL, 2013). Mesmo assim foi orientada a seguir consultando na UBS com agendamentos garantidos. 
Após algum tempo de perda do contato da gestante com a UBS, realizou-se sua busca ativa domiciliar, verificando que a paciente não estava realizando o acompanhamento pré-natal por não ter sido bem acolhida no serviço especializado e por não identificar mais a APS como local no qual deveria ser acompanhada. Acolhida novamente na UBS após convencimento da necessidade de realizar as consultas continuou fazendo acompanhamento de PN em nosso serviço com apoio matricial da equipe por uma médica endocrinologista do quadro de professores da UFPel.

\section{EVOLUÇÃO E DISCUSSÃO}

\section{Caso 1}

Romualdo iniciou contato com os cuidados da UBS, segundo seu prontuário, em meados de 1988, tendo estabelecido boa identificação com este serviço de saúde. Nota-se um discurso de desejo em seguir os planos terapêuticos propostos por parte do usuário, tanto no caráter farmacológico, quanto no não farmacológico, desde as primeiras anotações, demonstrando sua confiança no serviço, conforme predito na longitunalidade do cuidado. Segundo Starfield (2002), está é a "atenção continuada, com utilização do serviço pelo usuário ao longo do tempo, demonstrando e dependendo de uma relação de confiança entre profissionais de saúde e usuário" (SIRENA, 2016).

Durante seus 28 anos de relação com o serviço de saúde, usouse sempre uma abordagem multidisciplinar com o paciente (STARFIELD, 2002; MATTA, 2009), tendo documentação de consultas com a nutrição desde os anos 90 . Em alguns atendimentos recebeu visita domiciliares da enfermagem para orientações e controle glicêmico e pressórico, e da farmácia para organização dos treze medicamentos que tomava naquele momento (2016). Apesar da boa relação que pareceu haver entre o serviço de saúde e o paciente, houve muito pouca modificação no processo de evolução de seu quadro metabólico: seus valores pressóricos estiveram controlados apenas no início do quadro de HAS, seu perfil lipídico sempre esteve fora dos alvos terapêuticos, assim como seus níveis glicêmicos e de hemoglobina glicosilada (BRASIL, 2014; BRASIL, 2014a; BRASIL, 2014b). Não obstante, isso não significa que a relação do paciente com nosso serviço foi sem finalidade: o paciente já havia estado com mais peso e maior descontrole de tais níveis tensionais, notando que a intervenção multidisciplinar foi benéfica (BRASIL, 2014; BRASIL, 2014a; BRASIL, 2014b; BRASIL, 2014c).

Além disso, durante nosso início de contato em 2016, identificaram-se queixas que, embora menos graves, incomodavam-Ihe no dia-a-dia, como uma infecção micótica de pele recorrente retroauricular presente de forma intermitente há dois ou três anos e um quadro agudo de gota. Esse 
olhar ampliado pressupõe a abordagem clínica centrada na pessoa, uma vez que, sem minimizar outros aspectos da saúde de Romualdo, possivelmente mais mórbidos sob o ponto de vista da equipe de saúde, atentou-se também para aqueles que ele considerava importante, possibilitando melhor vínculo com o serviço e resolutividade/atenuação dos problemas (BRASIL, 2004; LOPES, 2012; FUZIKAWA, 2013). Estes problemas foram avaliados e manejados de forma interdisciplinar - atuação conjunta da equipe de enfermagem, medicina e farmácia. Podemos exemplificar como a equipe lidou com a situação uma vez que Romualdo fazia uso de forma inadequada do creme para a micose cutânea: após ida à residência do paciente, visualizando o uso do tópico do medicamento e reorientando-o é que tal terapêutica obteve sucesso. Isto demonstra a relevância de uma abordagem múltipla e integrada com estabelecimento de um projeto terapêutico específico e singular (STARFIELD, 2002; BRASIL 2012; BRASIL, 2014).

Infelizmente, apesar doa avanços alcançados pela equipe de APS não foi possível dar resposta a algumas demandas do usuário consideradas de maior complexidade (STARFIELD, 2002). Nos casos de necessidade de encaminhamento para a atenção secundária, este foi realizado por meio de contato direto com o serviço especializado da Faculdade de Medicina, um acesso só possível por os dois serviços serem da rede UFPel, mas o que não é realidade para todos os pacientes do sistema de saúde ou por meio do fluxo rotineiro via Secretaria Municipal de Saúde (extremamente demorado). Independente da forma de encaminhamento, estudos mostram que existem vários problemas de acesso a serviços especializados e, mesmo quando o usuário consegue o atendimento para o qual foi referenciado, não há a contrarreferência à APS (SPEDO et al., 2010; GIOVANELLA, 2012). Considerando integralidade os "serviços prestados que interferem com objetivo de fornecer integralmente saúde ao indivíduo, desde o processo de saúde-adoecimento até os serviços de média e alta complexidade", isso caracteriza um acesso à saúde irreal, uma vez que não é disponível a todos os usuários da APS (STARFIELD, 2002; SPEDO, 2010; GIOVANELLA, 2012).

Ainda acerca do acesso integral a saúde e considerando que as ações inerentes à APS têm potencial de interferir no processo de adoecimento, o serviço foi insuficiente (STARFIELD, 2008). Não obstante, não devido à imperícia dos profissionais do serviço de saúde, mas por conta da condição socioeconômica em que o paciente insere-se, demonstrando o peso da determinação social no processo saúde-doença, ou seja, que as condições econômicas e sociais exercem um efeito importante sobre a saúde e a doença dos indivíduos (BUSS, 2007; BRASIL, 2014).

A exemplificar, a intervenção nutricional, da qual Romualdo beneficiar-se-ia (BRASIL, 2014; BRASIL, 2014a; BRASIL, 2014b; BRASIL, 2014c), era sempre bem recebida pelo mesmo e seguida durante algum período. Porém, com o passar do tempo, as medidas ficavam dispendiosas, apesar das tentativas de adaptações à realidade econômica da família. As compras realizadas para o mês com o benefício que ele e a esposa recebiam de suas 
aposentadorias eram feitas em único dia, por assim conseguirem adquirir os produtos por preços mais baixos (compras em comércio atacadista). Assim, em geral optavam por alimentos menos perecíveis. Dessa forma, comprar mantimentos múltiplas vezes tornava-se caro por conta do deslocamento e o comprar múltiplas vezes por conta do preço. Outro exemplo eram os momentos em que os filhos e os netos iam fazer as refeições com Romualdo e sua esposa, quando o paciente acabava preparando a refeição e alimentando-se depois dos outros, restando para sua alimentação, na maioria das vezes, apenas arroz e feijão.

Ademais, o seu baixo nível de educação formal muitas vezes dificultou a compreensão de forma mais plena de seu estado de saúde, servindo de barreira para a recepção das medidas preventivas, sejam primárias, secundárias ou terciárias (STARFIELD, 2008). Dessa forma, mesmo que as orientações fossem feitas com Romualdo e sua família, elas encontravam dificuldade de serem postas em prática por conta de questões sociais e/ou econômicas (BUSS, 2017). Não obstante, a prevenção quaternária, neste caso, era factível e foi empregada, uma vez que a relação do serviço com o paciente através do acompanhamento longitudinal era bem estabelecida e evitou tanto a solicitação inadequada de exames de apoio diagnóstico quanto de encaminhamentos desnecessários (STARFIELD, 2008; GIOVANELLA, 2012).

Caso 2

Após busca ativa da paciente no seu domicílio por não comparecimento à consulta previamente agendada, ela retornou à UBS, ainda não para realização de atendimento $\mathrm{PN}$, mas como forma de ser reacolhida e recriar o vínculo com a equipe da UBS, de acordo com os preceitos da APS (STARFIELD, 2002). Inicialmente, o encontro não foi cordial, mas se conseguiu iniciar o processo para recriar o laço perdido e traçar-se um plano terapêutico conjunto, que levasse em consideração as expectativas e desejos da paciente (LOPES, 2012; FUZIKAWA, 2013).

A paciente mostrou-se certa de não querer retornar ao acompanhamento especializado, preferindo fazê-lo na UBS, demonstrando a dificuldade de concretizar-se o cuidado integral, apesar de ter conseguido acesso à atenção especializada (STARFIELD, 2002; BRASIL, 2010a). Contudo, nesse momento, criava-se uma encruzilhada, uma vez que se fazia necessário o apoio especializado para o bem-estar da gestante e de seu concepto (BRASIL, 2010; BRASIL, 2013).

Após o manejo feito pela equipe com Nize e o contato com os profissionais da atenção especializada, acordou-se que ela faria o acompanhamento apenas com a endocrinologista, não com todo o serviço de alto risco, demonstrando a qualidade na gestão da saúde desta usuária (STARFIELD, 2002; MATTA, 2009). Ainda que não ideal, a coordenação do cuidado possibilitou que a paciente realizasse seu pré-natal da forma mais adequada possível, surtindo resultados positivos, como o controle 
dos níveis glicêmicos e a ausência de complicações tangíveis à atenção primária ou secundária (BRASIL, 2010a; BRASIL, 2013).

O vínculo que se reestabeleceu mostrou-se tão importante e de tamanha confiança que, em uma das consultas, Nize foi ao serviço com as orientações da endocrinologista para saber se deveria ou não segui-las, o que demonstra o quanto, muitas vezes, o maior dos conhecimentos técnicos não é capaz de ajudar os pacientes sem uma adequada relação médico-paciente, ou, no caso, serviço-paciente (LOPES, 2012). O exposto reforça que a prática de acordo com o método clínico centrado na pessoa gera maior satisfação ao usuário e aos profissionais de saúde; melhora a adesão aos tratamentos propostos; e aumenta a recuperação de problemas recorrentes (LOPES, 2012; FUZIKAWA, 2013).

Dessa forma, o processo iniciado pela busca da paciente, a recuperação do vínculo entre Nize e o serviço e passando pela coordenação de seu cuidado gerou meios, tanto para o exercício do método clínico centrado na pessoa e da integralidade da atenção, quanto a materialização do atributo da longitudinalidade (STARFIELD 2002; MATTA, 2009; ORGANIZAÇÃO DAS NAÇÕES UNIDAS, 2009; LOPEZ, 2012; FUZIKAWA, 2013; BRASIL, 2013). Não obstante, há limitações no caso apresentado, uma vez que a paciente permaneceu tabagista, apesar de ter diminuído sua carga tabágica. Ademais, Nize reiniciou o uso de Fluoxetina por conta própria e manteve seu uso apesar de a equipe assistente desencorajar, já que a retomada foi motivada por sintomas ansiosos e depressivos leves, cujos riscos do medicamento não sobreporiam os benefícios, de acordo com o julgamento clínico da equipe na situação (BRASIL, 2010a).

\section{CONSIDERAÇÕES FINAIS}

Ao fim da análise, creio que tenhamos aplicado e vivenciado os princípios da Atenção Primária à Saúde, de forma a proporcionar um cuidado integral e qualificado aos pacientes, correspondendo aos preceitos da DUDH. Isso foi possível graças ao suporte de uma UBS com adequados recursos humanos e infraestrutura.

Entretanto, alguns pontos foram menos abarcados durante a prática do estágio. Devido ao grande volume de demanda espontânea, as ações programáticas da Estratégia de Saúde da Família - o modelo da APS brasileira -, eram de difícil gerenciamento. A busca ativa de pacientes faltosos, as visitas domiciliares, a atenção ao hipertenso, ao idoso, ao diabético, entre outros, foi menos realizada, ainda que presentes. Dessa forma, as orientações à família e, em especial, para a comunidade foram princípios menos presentes na vivência.

Além dos fatores tangíveis à UBS, a prática diária é bastante desafiadora quanto a viabilizar a integralidade para os usuários, em especial no acesso aos serviços de maior complexidade, de atenção secundária e a medicamentos mais caros. Esses impasses consumiam várias horas do diaa-dia dos profissionais e graduandos, visando o pleno direito do usuário à 
saúde.

Em suma, os princípios da APS foram respeitados e postos em prática. Isso possibilitou um atendimento multiprofissional de qualidade para os usuários, fazendo-os ter acesso a um serviço de saúde que visava praticar os preceitos da APS de acordo com o pregado DUDH, apesar dos limites logísticos da unidade.

\section{REFERÊNCIAS}

BRASIL. Constituição da República. Artigos 194, 196. Brasília: Senado Federal, 1988. Disponível em: <http://www.senado.gov.br/atividade/const/ con1988/CON1988_05.10.1988/CON1988.asp>. Acesso em: 30 mar. 2017.

BRASIL. Ministério da Saúde. Humaniza SUS: política nacional de humanização. A humanização como eixo norteador das práticas de atenção e gestão em todas as instâncias do SUS. Brasília: Ministério da Saúde, 2004. (Série B. Textos básicos de saúde).

. Manual do instrumento de avaliação da atenção primária à saúde: primary care assessment tool PCATool - Brasil. Brasília: Ministério da Saúde, 2010. (Série A. Normas e Manuais Técnicos).

. Manual técnico gestação de alto risco. Brasília: Ministério da Saúde, 2010a. (Série A. Normas e Manuais Técnicos. Cadernos de Atenção Primária n. 35).

Política Nacional de Atenção Básica. Brasília: Ministério da Saúde, 2012. (Série E. Legislação em saúde).

Atenção ao pré-natal de baixo risco. Brasília: Ministério da Saúde, 2013. (Série A. Normas e Manuais Técnicos. Cadernos de Atenção Primária n. 32).

Estratégias para o cuidado da pessoa com doença crônica. Brasília: Ministério da Saúde, 2014. (Série A. Normas e Manuais Técnicos. Cadernos de Atenção Primária n. 35).

Estratégias para o cuidado da pessoa com doença crônica diabetes mellitus. Brasília: Ministério da Saúde, 2014a. (Série A. Normas e Manuais Técnicos. Cadernos de Atenção Primária n. 36).

Estratégias para o cuidado da pessoa com doença crônica hipertensão arterial crônica. Brasília: Ministério da Saúde, 2014b. (Série A. Normas e Manuais Técnicos. Cadernos de Atenção Primária n. 37).

. Estratégias para o cuidado da pessoa com doença crônica obe-

sidade. Brasília: Ministério da Saúde, 2014c. (Série A. Normas e Manuais 
Técnicos. Cadernos de Atenção Primária n. 38).

BUSS, P. M.; FILHO, A. P. A saúde e seus determinantes sociais. Revista de Saúde Coletiva. Rio de Janeiro, v. 17, n. 1, p. 77-93, 2007.

CARVALHO, F. R. M. Os direitos humanos, a Declaração Universal dos Direitos Humanos de 1948 e o pensamento filosófico de Norberto Bobbio sobre os direitos do homem. Âmbito jurídico. Rio Grande. Disponível em: $<$ http://www.ambito-juridico.com.br/site/index.php?n_link=revista_artigos_ leitura\&artigo_id=5147>. Acesso em: 30 mar., 2017.

FACCHINI, L. A. et al. Avaliação de efetividade da Atenção Básica à Saúde em municípios das regiões Sul e Nordeste do Brasil: contribuições metodológicas. Caderno de Saúde Pública. Rio de Janeiro, v. 24, sup. 1, p. S159-S172, 2008.

FORTES, P. A. C. Ética, direitos dos usuários e políticas de humanização da atenção à saúde. Saúde e Sociedade. São Paulo, v. 13, n. 3, p. 30-35, 2004.

FUNDAÇÃO OSWALDO CRUZ. Saúde e direitos humanos. Ano 7. n. 7. Brasília: Fundação Oswaldo Cruz, 2010.

FUZIKAWA, A. K. O método clínico centrado na pessoa: um resumo. Belo Horizonte, [s. n.], 2013.

GIOVANELLA, L.; MENDONÇA, M. H. M. Atenção Primária à Saúde. In: GIOVANELLA, L. et al. (Orgs.). Políticas e sistema de saúde no Brasil. Rio de Janeiro, Ed. Fiocruz, 2012. p. 493-545.

LOPES, J. M. C. Consulta e abordagem centrada na pessoa. In: Tratado de medicina de família e comunidade [recurso eletrônico]: princípios, formação e prática. Orgs. Gusso, Gustavo, Lopes, José Mauro Ceratti. Porto Alegre: Artmed, p. 113-123, 2012. 2 v.

MATTA, G. C.; MOROSINI, M. V. G. Dicionário da educação profissional em saúde. Fundação Oswaldo Cruz. Escola Politécnica de Saúde Joaquim Venâncio. Rio de Janeiro, 2009. Disponível em: <http://www.epsjv.fiocruz. br/dicionario/verbetes/ateprisau.html>. Acesso: em 30 mar. 2017. Verbete consultado: Atenção primária à saúde.

ORGANIZAÇÃO DAS NAÇÕES UNIDAS. Centro de informações das nações unidas. Declaração universal dos direitos humanos. Rio de Janeiro: UNIC Rio, 2009.

RAMOS et. al. Polifarmácia e polimorbidade em idosos no Brasil: um desafio em saúde pública. Revista de Saúde Pública, n. 50, supl. 2, p. 9s, 2016.

ROCHA, A. C. D. et al. Atenção básica à saúde: avaliação de estrutura e 
processo. Revista de Administração em Saúde. São Paulo, v. 14, n. 54, p. 71-79, 2012.

SIRENA, S. A.; TARGA, L. V. Atenção primária à saúde: fundamentos para prática. Caxias do Sul: Educs, 2016.

SPEDO, S. M. et. al. O difícil acesso a serviços de média complexidade do SUS: o caso da cidade de São Paulo, Brasil. Revista de Saúde Coletiva, Rio de Janeiro, v. 20, n. 3, p. 953-972, 2010.

STARFIELD, B. Atenção primária: equilíbrio entre necessidades de saúde, serviços e tecnologia. Brasília: UNESCO; Ministério da Saúde, 2002.

STARFIELD, B. et al. The concept of prevention: a good idea gone astray? J. Epidemiol. Community Health. [England], v. 62, n. 7, p. 580-583, 2008.

Data de recebimento: 30 de março de 2017.

Data de aceite para publicação: 13 de junho de 2017. 